\title{
Free-ranging livestock and a diverse landscape structure increase bat foraging in mountainous landscapes
}

\author{
L. Ancillotto $\cdot$ F. Festa $\cdot$ F. De Benedetta $\cdot$ F. Cosentino $\cdot$ B. Pejic $\cdot$ \\ D. Russo
}

Received: 21 October 2020 / Accepted: 2 January 2021 / Published online: 10 January 2021

(C) The Author(s) 2021

\begin{abstract}
Traditional farming, where livestock is seasonally managed as free ranging and the use of drugs is reduced or absent, may prove beneficial to biodiversity by fostering the occurrence of spatial heterogeneity, and increasing the availability of trophic resources to wildlife. Previous work indicates that the presence of cattle in lowlands leads to an increase in bat foraging activity, yet no study has addressed this topic in mountainous regions, where free-ranging livestock is still common. Here we explore the relationships between landscape structure, farming and bat activity in a mountainous agricultural area, hypothesizing that bat activity will increase in
\end{abstract}

L. Ancillotto and F. Festa: Co-first authors.

Supplementary Information The online version contains supplementary material available at (https://doi.org/10.1007/ s10457-021-00591-0).

\footnotetext{
L. Ancillotto · D. Russo ( $\square)$

Wildlife Research Unit, Dipartimento di Agraria, Università degli Studi di Napoli Federico II, via Università 100, 80055, Portici (Napoli), Italy

e-mail: danrusso@unina.it

F. Festa $\cdot$ F. Cosentino

Dipartimento di Biologia e Biotecnologie "Charles

Darwin", Università degli Studi di Roma "La Sapienza",

Rome, Italy

F. De Benedetta

National Research Council (CNR), Institute for

Sustainable Plant Protection, 80055 Portici (Napoli), Italy
}

response to the presence of livestock and landscape structure and heterogeneity. We found that traditional cattle farming may have a role in influencing bat activity in mountainous agroecosystems, yet its effects are evident for a limited number of species. Three pipistrelle species favoured foraging in areas subjected to cattle farming by hunting more often over cattle or fresh dung than at control sites. Free-ranging cattle thus provide profitable foraging opportunities for bats in mountainous landscapes, which remarks the importance of traditional farming activities in sustaining biodiversity. Cattle might also benefit from bat foraging activity if this leads to suppression of bloodsucking pests.

Keywords Agroecosystems · Bats · Cattle · Farming $\cdot$ Livestock $\cdot$ Mountain ecology

\footnotetext{
B. Pejic

Department of Genetic Research, Institute for Biological Research "Siniša Stanković", National Institute of Republic of Serbia, University of Belgrade, Belgrade, Serbia

D. Russo

School of Biological Sciences, University of Bristol, 24 Tyndall Avenue, Bristol BS81TQ, UK
} 


\section{Introduction}

Progressive intensification of human activities globally altered the natural environment, and due to the need of natural resources, primary habitats have been replaced with agricultural systems (Park 2015). The unsustainable development of these practices gradually leads to an impoverishment of biological communities (Pereira et al. 2010). Negative effects of intensive livestock farming and agriculture on wildlife populations are multiple and diverse (Benton et al. 2003), depending on both the extent and magnitude of habitat grazed and the amount of managed landscape (Alkemade et al. 2013). Conversely, non-intensive farming practices, and the reduced use of livestock drugs, may prove beneficial to biodiversity by fostering the occurrence of spatial heterogeneity (Wickramasinghe et al. 2003), and increasing trophic resources (Mcadam et al. 2007). Several Mediterranean mosaic and semi-open habitats depend on human activities, including free-ranging livestock farming (Gonçalves et al. 2012). Altitude and terrain morphology also play an important role in shaping current and future trends in agricultural practices, with contrasting directions between low and high-elevation areas. While lowland systems tend towards intensification and biodiversity loss, mountainous areas, which used to feature non-intensive and traditional farming practices, are being abandoned in response to their lower productivity (Sokos et al. 2013).

Bats represent a successful taxonomic group in many human-modified landscapes worldwide, with many species occurring in artificial habitats (Wickramasinghe et al. 2003; Santini et al. 2019). Being multihabitat specialists and long-lived organisms, many bat species respond to even subtle habitat modifications, so that bats have been often addressed as excellent taxa for bioindication (Jones et al. 2009; De Conno et al. 2018). Habitat features used by bats range from highresolution landscape elements such as isolated trees and tree lines, hedges and water bodies, to large-scale characteristics such as connectivity between habitat patches, and habitat type distribution or availability over wide areas (Heim et al. 2015). Linear structures within a landscape enable bats to functionally locate themselves and navigate, e. g. to commute between roosts and foraging areas (Heim et al. 2015), acting as landmarks within the landscape matrix (Limpens and Kapteyn 1991).
The way bats use the landscape is deeply connected to their ecomorphology, e. g. echolocation call design, wing shape and size (Norberg and Rainer 1987; Russo et al. 2018a). Lower echolocation call frequencies and high flight speed are the best suite for bats that hunt in open areas (e.g. Voigt and Holderied 2012), while higher frequency echolocation calls and low flight speed are more effective in clutter habitats such as forests (Boyles et al. 2011). Bat foraging activity is generally lower in open landscapes because of the reduced concentration of insects in such habitats in comparison to tree canopy, or riparian habitats (Ancillotto et al. 2019), and because of the higher exposure to predators perceived by bats flying in open space (Russo et al. 2007). Thanks to the behavioural plasticity that characterizes most species, bats can exploit heterogeneity in landscape structure and composition: for example, forest bat species benefit from forest gaps because forest edges usually feature high densities of swarming insects compared to open areas and inner forest (Crome and Richards 1988). Creating and supporting spots of open areas, such as small-scale grazed areas in a wooded matrix, may thus enhance the presence of species related to complex mosaic habitats, increasing diversity within bat assemblages (Morris et al. 2010).

European bats are mostly insectivorous and provide important ecosystem services by suppressing herbivorous insects in forests (Böhm et al. 2011) and agricultural ecosystems (Boyles et al. 2011; Russo et al. 2018b). Besides, Downs and Sanderson (2010) and Ancillotto et al. (2017) proposed that an important attractive for bats in pastoral areas may be represented by blood-feeding pests parasitizing cattle, setting the potential for a new service provided by bats as cattle pest suppressors. The presence of cattle-related structures such as traditional stables may also have an underestimated importance to bats. Many species base their diets on flies and spiders gleaned from the substrate (Krull et al. 1991), and both prey types may occur in high numbers inside cattle stables (Siemers et al. 2012), which thus represent a potential foraging habitat. Despite their importance, bats have long been unnoticed ecosystem service providers, thus the effects of landscape changes on such services is still poorly understood, and the ecological knowledge to promote bats in agroecosystems needs further investigation (Froidevaux et al. 2019). 
Here we explore the relationships between landscape structure, livestock farming and bat activity in a mountainous agricultural area of central Italy. Specifically, we hypothesize that bat activity will respond to the presence of free-ranging livestock and to landscape composition and configuration in a forestpasture mosaic landscape. We predict that bats will increase activity near cattle and in areas with larger amounts of forest and linear landscape elements, showing species- and scale-specific responses.

\section{Materials and methods}

Study area

Our study was conducted in the Abruzzo, Lazio and Molise National Park ( $41^{\circ} 48^{\prime} 30.2^{\prime \prime} \mathrm{N}, 13^{\circ} 47^{\prime} 11.3^{\prime \prime}$ E). The landscape within the study area encompassed several habitat types, including mountainous areas with high altitude grasslands and rocky areas, beech forests (Fagus sylvatica L.), villages, farms and extensive agricultural systems (Fig. 1).

\section{Bat activity surveys}

We identified 15 sampling areas within the Park and its immediate surroundings at altitudes of 900-1520 m a.s.l. The landscape is dominated by a mosaic of beech forest patches interspersed within a matrix of pastures, regularly grazed by cattle, while forest patches are used by livestock to recover at night. The cattle in the area are traditionally managed by alternating indoor recovery during winter in low altitude farms, and a free-ranging phase in mountain pastures throughout summer. Within each of these 15 areas, following Ancillotto et al. (2017), we located three recording sites, each assigned to one of three categorical classes, referring to cattle presence at the time of the recording (summarized as "treatment" from here on):

- cattle present; cattle were present within a distance of 5-15 m from the recorder; cattle were either grazing or sleeping, and the numbers of head were recorded at the start of the recording session and entered in statistical models as a nested covariate;

- fresh dung: fresh cattle dung was present in the immediate surroundings ( $0-2 \mathrm{~m}$ ) of the recorder, yet cattle was not present within a range of $100 \mathrm{~m}$; dung was considered fresh when it was not dried, and emergence holes by dung-feeding insects were not yet present;

- control: neither fresh dung nor cattle were present within $100 \mathrm{~m}$ from the recorder.

Sampling areas were $4.5 \pm 3.7 \mathrm{~km}$ (range: 1.1-10.2) apart, and recording sites within each area were $179.5 \pm 56.2 \mathrm{~m}$ apart (range: $150-310 \mathrm{~m}$ ), to avoid multiple counts of the same bat at more recording sites. Sites within the same area were always sampled simultaneously on the same night to exclude any effect of temporal variation in local activity levels.

We used Pettersson D1000x bat detectors (Pettersson Elektronik AB) and recorded bat activity for $4 \mathrm{~h}$ after sunset from June to August 2017. The detectors were operated manually in the heterodyne mode and switched continuously between 20 and $110 \mathrm{kHz}$ ca. to cover all bat species present in the area. When a bat was detected, the real-time recording was activated and the signals were recorded at a sampling rate of $380 \mathrm{kHz}$, and saved onto $\mathrm{CF}$ cards.

Sound analysis was made using BatSound 4.2; for every bat pass, 3 calls were selected to manually measure spectral and temporal variables, and identification was carried out following Russo and Jones (2002) and using reference calls. Identification was performed to species level except for those species showing a high overlap among echolocation call parameters, which were pooled and classified as Myotis spp. and Nyctalus spp., respectively. Similarly, calls classified as Pipistrellus kuhlii may have also included some $P$. nathusii, yet the latter was only captured once in $>10$ years of mistnetting in the study area, thus its presence is very rare. For each recording night/site we measured the total number of bat passes, numbers of passes per taxon, and numbers of feeding buzzes (i.e. call sequences emitted by bats attempting prey capture; Griffin et al. 1960; Russo et al. 2016), both total and by taxon.

\section{Land use mapping}

Land use was mapped using Corine land cover 2012 layers (Open data, GeoportaleNazionale.org), reclassified into two habitat-type classes representative of the dominant habitat type, i.e. agricultural land and forest, cumulatively representing $>95 \%$ of land use 

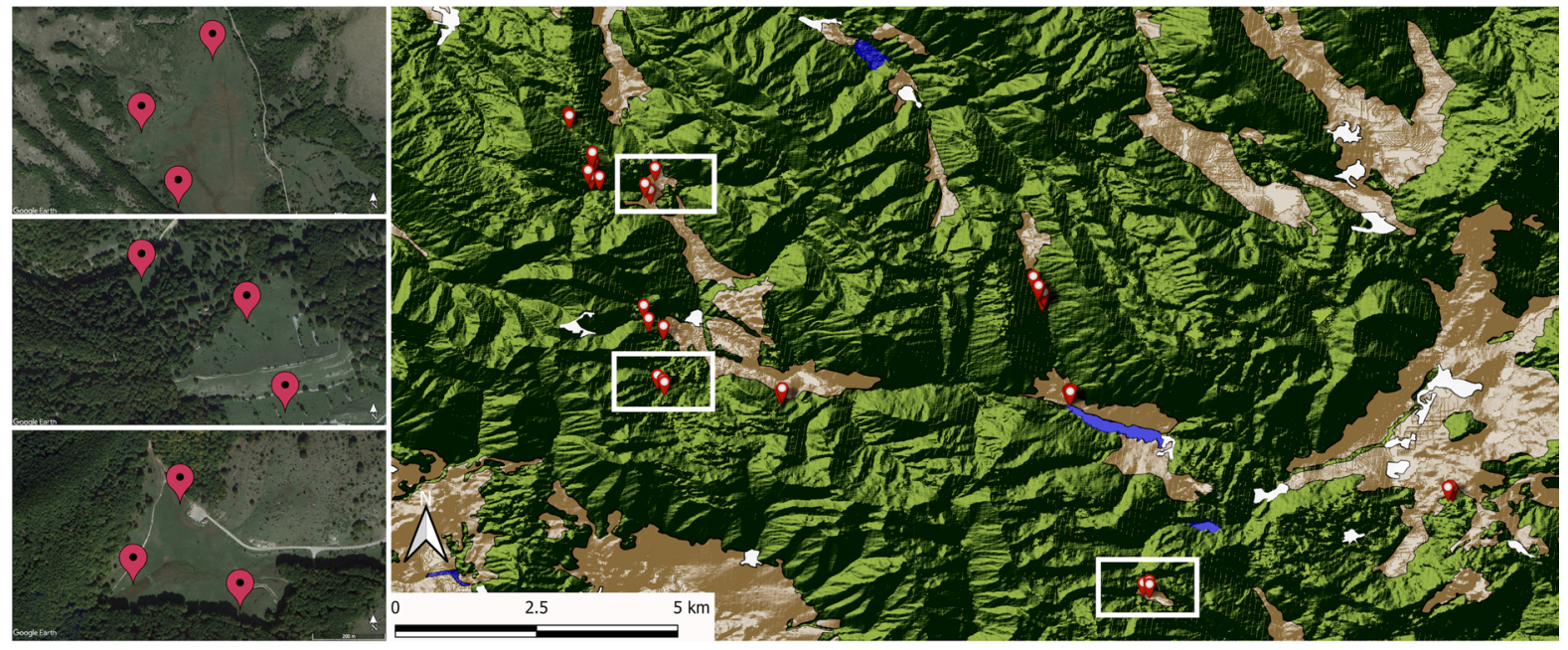

Fig. 1 Bat recording sites (red placemarks) in the Abruzzo Lazio and Molise National Park (Central Italy) and the main habitat types (in different colours). Shades indicate altitude and slope green $=$ wooded areas; brown $=$ pastures and agricultural fields; white = urban areas; blue $=$ lakes. Inserts to the left are satellite images showing the exact location of recording sites

in the study area. Information about landscape composition and configuration was extracted from land use maps in QGis (https://www.qgis.org/it/site/) through the LecoS plugin (http://plugins.qgis.org/plugins/ LecoS/) as the percent amount of forest, number of forest patches, and amount of edge habitat (expressed in $\mathrm{m}$ per ha). The latter was calculated following Jung (2013), and quantifies the amount of edge segments involving forest patches; therefore, we used it as a proxy of available edge habitat at each sampling point. Each landscape descriptor was independently calculated at three scales for each site $(n=45)$ by extracting the information within circular "landscapes" of different sizes, i.e. circles characterized by radiuses of 50 , 100 and $300 \mathrm{~m}$ respectively around a given site. These spatial scales are representative of the size of bat foraging sites (Bellamy et al. 2013) and are usually adopted for this kind of studies; additionally, altitude was also recorded for each site from GPS data (using a Garmin Dakota10, 3 m accuracy).

Statistical analyses

To measure the response of bats to landscape structure and treatment, we adopted generalized linear mixed models (GLMM, lmer function - R package lme4Bates et al. 2020) using binomial negative error (red placemarks, corresponding to three treatments: cattle; dung; and control) within the three sampling areas evidenced by white rectangles in the main image. Corine Land Cover and Digital Elevation Model layers were taken from GeoportaleNazionale.org; satellite images from Google Earth Pro 7.3, downloaded on November 24th 2020

distribution and a log link function. We then built three separate models for each response variable corresponding to the spatial scales considered. We used landscape descriptors, treatment and altitude as explaining variables. Data were all log-transformed before analyses to meet model assumptions. We assessed variable collinearity by selecting only those with a Spearman's correlation coefficient between 0.80 and 0.80 , and a variance inflation factor (VIF) $<10$ (Zuur et al. 2010). We tested whether the numbers of bat passes were positively associated with the numbers of feeding buzzes by running a Pearson's correlation test. In all models, we accounted for potential confounding effects due to recording at three sites in each area using area identity as a random factor.

We assessed single-variable importance in explaining bat responses following a likelihood ratio approach achieved by applying the built-in drop1 and ANOVA functions in $\mathrm{R}$ to compare each full model with that lacking a given variable. We considered significant the effect of a given variable when ANOVA's $p<0.05$. When the treatment variable showed a significant effect on model performance, we applied a Tukey's post-hoc test to establish differences among treatment levels (glht function- R package multcomp_Bretz et al. 2020). 


\section{Results}

We recorded 1,263 passes and 73 feeding buzzes of 14 bat species/species groups, with the most common species at all sites being Pipistrellus kuhlii/P. nathusii, which made up for $28 \%$ of the total acoustic sample (table S1 in Supporting Information). Numbers of feeding buzzes showed a strong positive correlation with bat activity (Pearson's $r=0.71, p<0.01$ ), thus we used the numbers of passes as a genuine proxy of bat foraging activity levels.

Cattle treatment had a significant effect on the activity of $P$. pipistrellus (all scales), $H$. savii and $P$. kuhlii (100 m scales). The three species were generally more active over dung and cattle than at control sites (Fig. 2), yet differed in their responses. P. pipistrellus and $H$. savii showed significantly higher activity over dung than at control and cattle sites (both Tukey's $p<0.05$ ), respectively. Despite the GLMM showed a significant difference for $P$. kuhlii/nathusii, posthoc tests failed to detect significant differences among treatments for this taxon (Fig. 2). Nyctalus spp. did not respond to any considered variable, but was more active over dung than in other treatments (figure $\mathrm{S} 1$ in Supporting Information). The numbers of heads per herd (mean: $55 \pm 60$, range: 5-350) had a positive effect on bat activity only for $P$. kuhlii/nathusii and negative for $P$. pipistrellus at the $50 \mathrm{~m}$-scale.

The presence and degree of interspersion of wooded areas enhanced total bat activity, with a significant positive effect of the percentage of wooded area at $50 \mathrm{~m}$ and $100 \mathrm{~m}$ scales. Forest patchiness had a significantly negative effect at $100 \mathrm{~m}$, and a positive effect at $300 \mathrm{~m}$ from the recording site (Fig. 3). Landscape configuration and structure both proved to be important drivers of bat activity for most species considered (Table 1). Edge density showed a positive effect on Hypsugo savii and P. pipistrellus at $50 \mathrm{~m}$ and $100 \mathrm{~m}$ scales. The percent amount of wooded areas had a significant and positive effect on $P$. kuhlii and Tadarida teniotis only at the $50 \mathrm{~m}$ scale. The numbers of forest patches influenced positively activity of $T$. teniotis only at $50 \mathrm{~m}$ and P. kuhlii only at $300 \mathrm{~m}$. Site altitude also played a significant role by enhancing the activity of $P$. pipistrellus (50 $\mathrm{m}$ and $30 \mathrm{~m}$ scales) and Myotis bats (300 m scale).

\section{Discussion}

In agreement with our hypotheses and with previous studies in different ecological contexts, we found that traditional cattle farming plays a role in influencing the use of space by bats in mountainous agroecosystems. The main evidence we found was that three small species from the genera Pipistrellus and Hypsugo favour areas subjected to cattle farming, specifically by hunting more intensively over cattle or fresh dung. This result differs from what was shown in another study dealing with the same species, where the positive effect of cattle was recorded for more species
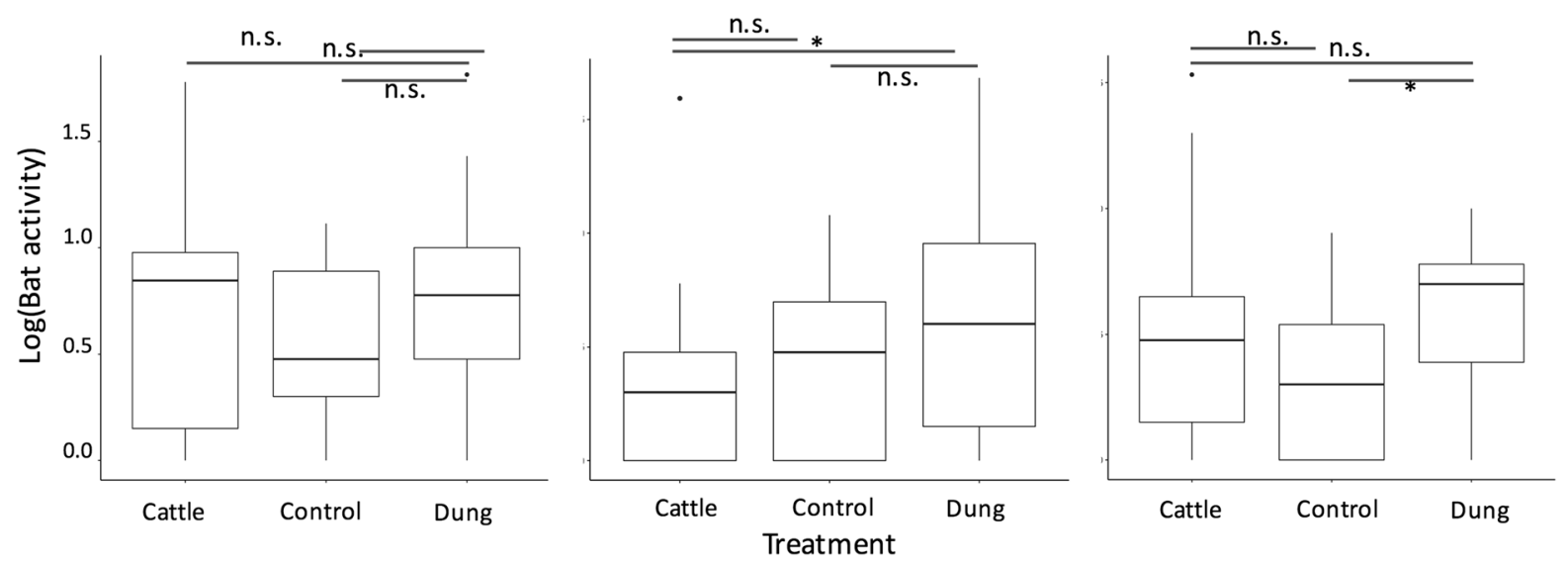

Fig. 2 Numbers of passes by three pipistrelle bats (left: Pipistrellus kuhlii; middle: Pipistrellus pipistrellus; right: Hypsugo savii) recorded in a forest/agricultural landscape of central Italy at 45 locations under three different treatments: Cattle $=$ cattle present at recording site; Dung $=$ fresh

dung (but no cattle) present; Control = neither cattle or dung present at recording site. Tukey's post-hoc significance of pairwise comparisons is indicated as: n.s. = non significant, $*=p<0.05$ 


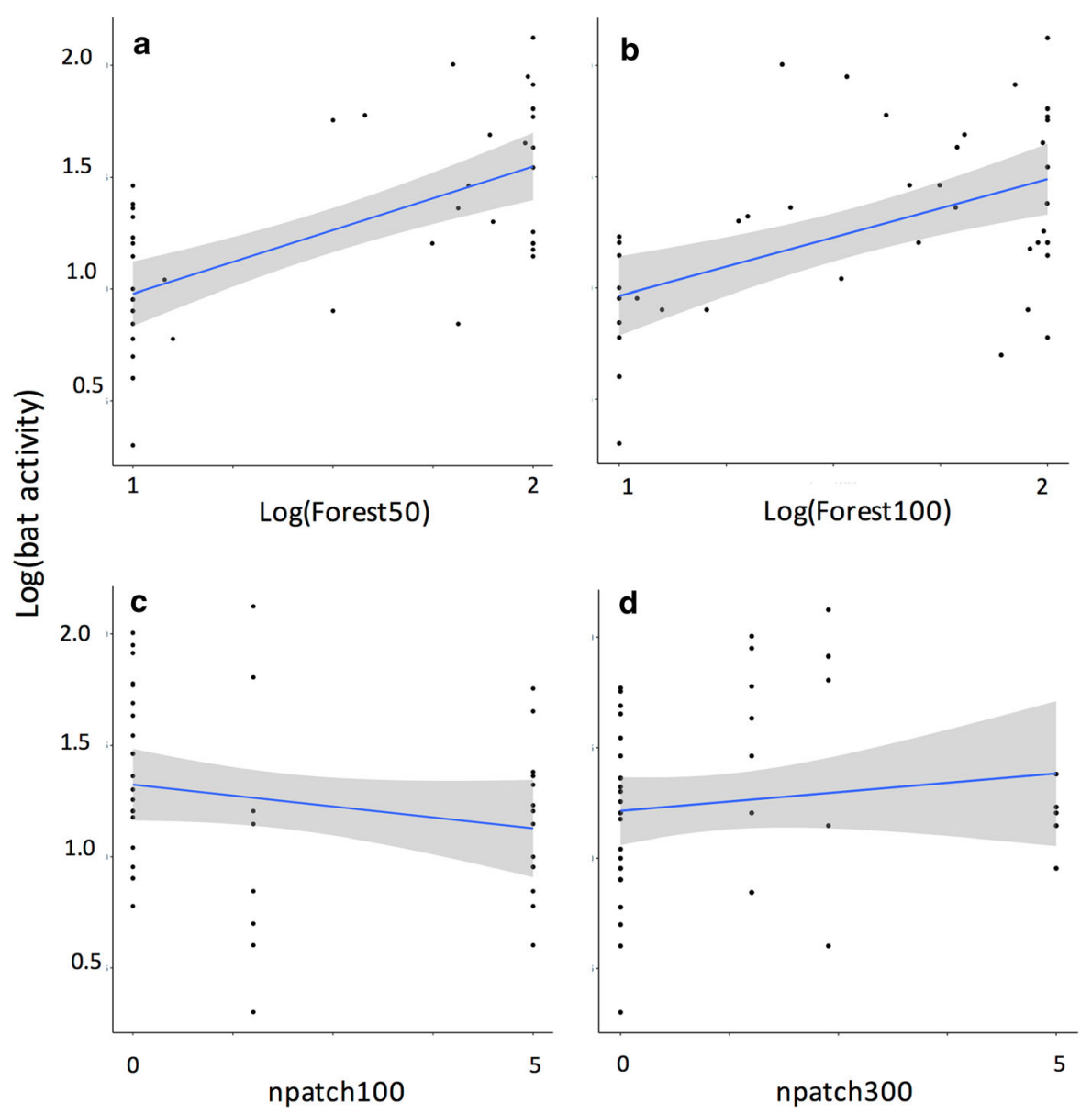

Fig. 3 Total bat activity in relation to the amount of forest within a radius of 50 (a) and 100 (b) $\mathrm{m}$ from the recorder, and to the number of forest patches within a radius of 100 (c) and 300 (d) $\mathrm{m}$ from the recorder in a mountainous landscape of central Italy $(\mathrm{n}=45)$

(Ancillotto et al. 2017). Such discrepancy may reflect the different altitudes of the two study areas (FreyEhrenbold et al. 2013). Elevations $\geq 900 \mathrm{~m}$ a.s.l. in temperate regions such as those in the present study may be unsuitable for many nocturnal blood-sucking dipterans (Roiz et al. 2011), and eventually make foraging over cattle herds by bats less profitable. Moreover, despite the two studies were based on similar sampling efforts and applied identical protocols, the activity levels recorded by Ancillotto et al. (2017) were $>3$ times higher. This result could represent a response to higher temperatures, which foster greater insect availability at lower altitudes (Georgiakakis et al. 2010; Grindal et al. 1999; Taylor 1963). Our sampling protocol did not allow to test long-term associations between bats and cattle at each site, yet the spatial and temporal scales of our study, covering an area of ca. 31,000 ha over 3 months, made the risk of detecting a coincidental association unlikely.

A mutualistic relationship between bats and large ungulates was first suggested by studies that acoustically surveyed bat activity near cattle (Downs and Sanderson 2010), and was also recently reported by photographic evidence on wild herbivores (Palmer et al. 2019), suggesting that this may be a far more frequent phenomenon than previously expected deserving further attention. The advent of agriculture in different geographical areas and the consequent replacement of herd of wild large herbivores by domestic ones which occurred throughout the Holocene (Hearn 2015) may have offered bats a unique opportunity to shift adaptively their mutualistic host and thus provide a novel ecosystem service to humans, i.e. cattle pest suppression. 
Table 1 Results of Generalized Linear Mixed Models testing the effects of cattle (Treatment, N Cows) and landscape characteristics on bat activity in a mountainous area of central Italy. Spatial scale indicates the radius (in $\mathrm{m}$ ) of the circular plot around the sampling point where the variables were measured. \%Forest $=$ percent amount of forest cover; $\mathrm{N}$ Patches = numbers of forest patches; Edge density = amount of habitat edges. **: $p<0.01 ; *: p<0.05$. Empty cells in the $P$ column indicate non-significant results

\begin{tabular}{|c|c|c|c|c|c|c|}
\hline Spatial scale & Response & Variable & Estimate & SE & $\mathrm{Z}$ & $p$ \\
\hline \multirow[t]{6}{*}{50} & \multirow[t]{6}{*}{ Activity } & Treatment & 9.07 & 9.58 & 3.02 & \\
\hline & & N Cows & 0.11 & 0.17 & 0.05 & \\
\hline & & Altitude & -0.91 & 1.35 & 0.38 & \\
\hline & & $\%$ Forest & 0.36 & 0.15 & 3.54 & * \\
\hline & & N Patches & -0.61 & 0.56 & 0.77 & \\
\hline & & Edge Density & -2.80 & 1.82 & 2.36 & \\
\hline \multirow[t]{6}{*}{100} & \multirow[t]{6}{*}{ Activity } & Treatment & 4.73 & 11.49 & 3.29 & \\
\hline & & N Cows & 0.19 & 0.17 & 0.08 & \\
\hline & & Altitude & -0.29 & 1.61 & 0.28 & \\
\hline & & $\%$ Forest & 0.60 & 0.26 & 0.91 & * \\
\hline & & N Patches & -2.30 & 1.02 & 3.57 & * \\
\hline & & Edge Density & -4.04 & 3.26 & 1.54 & \\
\hline \multirow[t]{6}{*}{300} & \multirow[t]{6}{*}{ Activity } & Treatment & 4.94 & 10.34 & 3.13 & \\
\hline & & N Cows & 0.02 & 0.17 & 0.05 & \\
\hline & & Altitude & -0.33 & 1.48 & 0.36 & \\
\hline & & $\%$ Forest & -0.27 & 0.18 & 0.20 & \\
\hline & & N Patches & 1.32 & 0.57 & 6.81 & * \\
\hline & & Edge Density & 160.35 & 435.38 & 0.13 & \\
\hline \multirow[t]{6}{*}{50} & \multirow[t]{6}{*}{ Hypsugo savii } & Treatment & -11.59 & 10.17 & 2.64 & \\
\hline & & N Cows & 0.27 & 0.22 & 1.30 & \\
\hline & & Altitude & 1.76 & 1.43 & 0.37 & \\
\hline & & $\%$ Forest & 0.34 & 0.19 & 0.04 & \\
\hline & & N Patches & -0.46 & 0.71 & 0.03 & \\
\hline & & Edge Density & -4.99 & 2.24 & 4.95 & $*$ \\
\hline \multirow[t]{6}{*}{100} & \multirow[t]{6}{*}{ Hypsugo savii } & Treatment & -16.76 & 10.87 & 2.77 & * \\
\hline & & N Cows & 0.27 & 0.22 & 1.31 & \\
\hline & & Altitude & 2.46 & 1.53 & 0.37 & \\
\hline & & $\%$ Forest & 0.43 & 0.31 & 0.03 & \\
\hline & & N Patches & -0.67 & 1.23 & 0.73 & \\
\hline & & Edge Density & -8.34 & 3.58 & 5.44 & * \\
\hline \multirow[t]{6}{*}{300} & \multirow[t]{6}{*}{ Hypsugo savii } & Treatment & 2.13 & 3.80 & 1.22 & \\
\hline & & N Cows & 0.04 & 0.082 & 0.45 & \\
\hline & & Altitude & -0.07 & 0.54 & 0.11 & \\
\hline & & $\%$ Forest & -0.04 & 0.07 & 0.03 & \\
\hline & & N Patches & 0.23 & 0.23 & 1.23 & \\
\hline & & Edge Density & 69.96 & 201.94 & 0.12 & \\
\hline \multirow[t]{6}{*}{50} & \multirow[t]{6}{*}{ Pipistrellus kuhlii } & Treatment & 15.50 & 11.38 & 1.29 & \\
\hline & & N Cows & 0.51 & 0.24 & 2.80 & $*$ \\
\hline & & Altitude & -2.03 & 1.60 & 0.89 & \\
\hline & & $\%$ Forest & 0.42 & 0.21 & 4.58 & $*$ \\
\hline & & N Patches & -0.69 & 0.79 & 0.46 & \\
\hline & & Edge Density & -2.33 & 2.50 & 0.86 & \\
\hline
\end{tabular}


Table 1 continued

\begin{tabular}{|c|c|c|c|c|c|c|}
\hline Spatial scale & Response & Variable & Estimate & SE & $\mathrm{Z}$ & $p$ \\
\hline \multirow[t]{6}{*}{100} & $\begin{array}{l}\text { Pipistrellus } \\
\quad \text { kuhlii/nathusii }\end{array}$ & Treatment & 13.58 & 12.96 & 1.20 & $*$ \\
\hline & & N Cows & 0.53 & 0.26 & 2.65 & \\
\hline & & Altitude & -1.76 & 1.82 & 0.78 & \\
\hline & & $\%$ Forest & 0.38 & 0.37 & 1.53 & \\
\hline & & N Patches & -1.16 & 1.47 & 0.55 & \\
\hline & & Edge Density & -1.32 & 4.26 & 0.10 & \\
\hline \multirow[t]{6}{*}{300} & $\begin{array}{l}\text { Pipistrellus } \\
\quad \text { kuhlii/nathusii }\end{array}$ & Treatment & 8.08 & 13.03 & 1.63 & \\
\hline & & N Cows & 0.42 & 0.23 & 3.97 & \\
\hline & & Altitude & -1.01 & 1.86 & 0.70 & \\
\hline & & $\%$ Forest & -0.29 & 0.24 & 1.03 & \\
\hline & & N Patches & 1.78 & 0.73 & 8.97 & $*$ \\
\hline & & Edge Density & 668.69 & 575.29 & 1.35 & \\
\hline \multirow[t]{6}{*}{50} & Pipistrellus pipistrellus & Treatment & 13.76 & 6.47 & 2.62 & $*$ \\
\hline & & N Cows & -0.35 & 0.18 & 4.99 & $*$ \\
\hline & & Altitude & -1.82 & 0.91 & 10.44 & $*$ \\
\hline & & $\%$ Forest & 0.20 & 0.16 & 0.23 & \\
\hline & & N Patches & 0.01 & 0.60 & 0.61 & \\
\hline & & Edge Density & -3.35 & 1.76 & 3.63 & $*$ \\
\hline \multirow[t]{6}{*}{100} & Pipipstrellus pipistrellus & Treatment & 10.28 & 6.79 & 2.72 & $*$ \\
\hline & & N Cows & -0.33 & 0.19 & 5.25 & \\
\hline & & Altitude & -1.35 & 0.96 & 10.96 & \\
\hline & & $\%$ Forest & 0.35 & 0.23 & 0.61 & \\
\hline & & N Patches & -0.72 & 0.93 & 0.40 & \\
\hline & & Edge Density & -5.57 & 2.43 & 5.24 & $*$ \\
\hline \multirow[t]{6}{*}{300} & Pipistrellus pipistrellus & Treatment & 19.99 & 7.48 & 2.94 & $* *$ \\
\hline & & N Cows & -0.33 & 0.19 & 3.59 & \\
\hline & & Altitude & -2.74 & 1.07 & 8.02 & $*$ \\
\hline & & $\%$ Forest & -0.06 & 0.15 & 0.74 & \\
\hline & & N Patches & 0.75 & 0.49 & 2.22 & \\
\hline & & Edge Density & -168.97 & 452.58 & 0.14 & \\
\hline \multirow[t]{6}{*}{50} & Myotis sp. & Treatment & -10.95 & 7.23 & 0.86 & \\
\hline & & N Cows & -0.14 & 0.19 & 0.38 & \\
\hline & & Altitude & 1.70 & 1.02 & 2.20 & \\
\hline & & $\%$ Forest & 0.26 & 0.17 & 0.00 & \\
\hline & & N Patches & -1.02 & 0.63 & 1.70 & \\
\hline & & Edge Density & -2.12 & 1.89 & 1.25 & \\
\hline \multirow[t]{6}{*}{100} & Myotis sp. & Treatment & -10.77 & 8.05 & 0.90 & \\
\hline & & N Cows & -0.09 & 0.20 & 0.35 & \\
\hline & & Altitude & 1.69 & 1.13 & 2.02 & \\
\hline & & $\%$ Forest & 0.21 & 0.26 & 0.87 & \\
\hline & & N Patches & -1.44 & 1.05 & 2.06 & \\
\hline & & Edge Density & -0.91 & 2.82 & 0.10 & \\
\hline 300 & Myotis sp. & Treatment & -12.45 & 7.10 & 0.79 & \\
\hline
\end{tabular}


Table 1 continued

\begin{tabular}{|c|c|c|c|c|c|c|}
\hline Spatial scale & Response & Variable & Estimate & SE & $\mathrm{Z}$ & $p$ \\
\hline & & N Cows & -0.18 & 0.21 & 0.40 & \\
\hline & & Altitude & 1.95 & 1.03 & 2.34 & $*$ \\
\hline & & $\%$ Forest & -0.09 & 0.15 & 1.19 & \\
\hline & & N Patches & -0.15 & 0.50 & 0.04 & \\
\hline & & Edge Density & 262.33 & 494.73 & 0.28 & \\
\hline \multirow[t]{6}{*}{50} & Tadarida teniotis & Treatment & 2.67 & 11.00 & 2.20 & \\
\hline & & $\mathrm{N}$ Cows & 0.04 & 0.12 & 0.03 & \\
\hline & & Altitude & -0.32 & 1.54 & 0.05 & \\
\hline & & $\%$ Forest & 0.19 & 0.10 & 0.63 & $*$ \\
\hline & & N Patches & -0.88 & 0.41 & 4.45 & $*$ \\
\hline & & Edge Density & -0.80 & 1.40 & 0.33 & \\
\hline \multirow[t]{6}{*}{100} & Tadarida teniotis & Treatment & 2.13 & 11.66 & 2.04 & \\
\hline & & N Cows & 0.04 & 0.14 & 0.03 & \\
\hline & & Altitude & -0.23 & 1.63 & 0.05 & \\
\hline & & $\%$ Forest & 0.02 & 0.22 & 0.44 & \\
\hline & & N Patches & -0.84 & 0.81 & 2.12 & \\
\hline & & Edge Density & 1.36 & 2.78 & 0.24 & \\
\hline \multirow[t]{6}{*}{300} & Tadarida teniotis & Treatment & $4.19 \mathrm{e}-01$ & $1.16 \mathrm{e}+01$ & 1.83 & \\
\hline & & N Cows & $1.35 \mathrm{e}-02$ & $1.38 \mathrm{e}-01$ & 0.03 & \\
\hline & & Altitude & $6.87 \mathrm{e}-04$ & $1.65 \mathrm{e}+00$ & 0.04 & \\
\hline & & $\%$ Forest & $-8 \mathrm{e}-02$ & $1.78 \mathrm{e}-01$ & 0.27 & \\
\hline & & N Patches & $1.39 \mathrm{e}-01$ & $5.27 \mathrm{e}-01$ & 0.00 & \\
\hline & & Edge Density & $-2.07 \mathrm{e}+02$ & $3.61 \mathrm{e}+02$ & 0.33 & \\
\hline \multirow[t]{6}{*}{50} & Nyctalus sp. & Treatment & 12.57 & 11.04 & 4.91 & \\
\hline & & N Cows & 0.13 & 0.18 & 0.37 & \\
\hline & & Altitude & -1.67 & 1.55 & 0.02 & \\
\hline & & $\%$ Forest & -0.05 & 0.15 & 3.56 & \\
\hline & & N Patches & 0.51 & 0.58 & 0.54 & \\
\hline & & Edge Density & 2.38 & 1.91 & 1.55 & \\
\hline \multirow[t]{6}{*}{100} & Nyctalus sp. & Treatment & 11.27 & 12.18 & 5.32 & \\
\hline & & N Cows & 0.18 & 0.18 & 0.38 & \\
\hline & & Altitude & -1.50 & 1.71 & 0.01 & \\
\hline & & $\%$ Forest & 0.28 & 0.27 & 6.01 & \\
\hline & & N Patches & -0.71 & 1.05 & 0.88 & \\
\hline & & Edge Density & 0.91 & 3.39 & 0.07 & \\
\hline \multirow[t]{6}{*}{300} & Nyctalus sp. & Treatment & 7.94 & 11.32 & 4.09 & \\
\hline & & N Cows & 0.15 & 0.19 & 0.34 & \\
\hline & & Altitude & -1.03 & 1.62 & 0.02 & \\
\hline & & $\%$ Forest & 0.07 & 0.20 & 0.71 & \\
\hline & & N Patches & 0.31 & 0.63 & 0.03 & \\
\hline & & Edge Density & -474.57 & 491.45 & 0.93 & \\
\hline
\end{tabular}

As we hypothesised, bat activity was also affected by landscape structure, including both composition and configuration. Even in structurally simple landscapes such as the one we examined, where land cover only comprised two main classes, the amount of forest cover played an important role in favouring bat 
activity. Forest habitats are a fundamental source of insects and thus provide important foraging opportunities to many bat species, as evidenced in a variety of ecosystems, from high mountain (Jaberg and Guisan 2001) to low elevation areas (Grindal and Brigham 1999), agricultural landscapes (Heim et al. 2015) and urban areas (Ancillotto et al. 2019). Dense and open canopy forests are favoured by a set of bat species occurring in European mountains, e.g. Myotis myotis and Eptesicus nilssoni in Switzerland (Jaberg and Guisan 2001), and P. pipistrellus and Nyctalus spp. in Germany (Kusch et al. 2004), as also confirmed by our study.

Landscape configuration is also known to affect the use of space by bats, influencing activity levels (Downs and Racey 2006). In our study, we evidenced that both forest patchiness in terms of numbers of distinct forest fragments, and the density of hedgerows, margins and tree-lines, played a major role in shaping bat activity, both at the entire assemblage and at single-species levels. Yet, forest patchiness showed diverging effects at different scales: small-scale patchiness negatively affected bat activity, while at larger distances it had a weak yet positive effect. Such divergence in the effect of patchiness may be due to a lack of easily identifiable landmarks such as margins where edge density is too high, making it more difficult for bats to detect and follow linear landmarks that favour commuting and foraging. Scale-dependent diverging effects of landscape configuration are already known for bats (Klingbeil and Willig 2009), as well as other highly mobile vertebrates (Fraser and Stutchbury 2004; Ferreira et al. 2018).

Bats from different ecomorphological guilds may respond differently to the presence of gaps within a forest (Crome and Richards 1988) or make a different use of linear elements, e.g. by avoiding to cross open spaces (Frey-Ehrenbold et al. 2013). The bat assemblage in our study was dominated by species characterised by medium-to-high wing aspect ratios, and thus capable of flying across open areas (Limpens and Kapteyn 1991). Thus, our results might not be extended to more strictly forest-adapted species such as those within the Myotis and Plecotus genera (Entwistle 1996).

Free-ranging cattle provided profitable foraging opportunities only for two small aerial-hawkers which due to their ecological flexibility may still cope with food paucity typical of high altitudes (Dunn and
Waters 2012). We are confident that the activity levels we measured reflect habitat preferences because (a) the species that are known to roost in the surroundings of our study sites were never or rarely recorded (e.g. Barbastella barbastellus: Russo et al. 2017a, b), (b) the most frequently recorded species (pipistrelles) only roosted in buildings in the study area, $>2 \mathrm{~km}$ away from our sites, and c) to our best knowledge, there were no buildings near the sampling sites hosting bat colonies.

The bat species we found to forage near cattle are those that prey on insects typically associated with livestock (dipterans: Culicidae and Simulidae; Standfast and Dyce 1968), and dung (coleopterans: Scarabeidae and Geotrupidae; Merritt and Anderson 1977). In mountain landscapes, forest gleaners too may still indirectly benefit from livestock farming by feeding on flies inside stables (Kervyn et al. 2012; Siemers et al. 2012). In the study area, we mistnetted Myotis mystacinus inside and near stables, suggesting that this species may represent another stable feeder (pers. obs.).

Bats are in most cases species of conservation concern due to their sensitivity to habitat changes, which make them vulnerable to a wide range of threats (Frick et al. 2019; Russo et al. 2017a, b). Our results suggest that maintaining low-intensity traditional farming in mountainous areas provide an asset to foster bat presence by offering profitable foraging conditions in otherwise relatively unproductive habitats (Lison et al. 2020), where interspecific competition among bat species in the same foraging guild is more likely to occur (Salinas-Ramos et al. 2020). Consequently, free-ranging and traditionally managed livestock should be seen as another management variable for bat conservation, in return for a potential ecosystem service provided by bats as pest suppressors.

Acknowledgements We thank the Abruzzo Lazio and Molise National Park and particularly Cinzia Sulli, head of the Park's scientific service, for authorizing and promoting this study. Claudio Di Domenico kindly allowed us to record bats near his livestock as well as on his property. We are grateful to Brock Fenton and two anonymous reviewers for providing important advice that helped us to improve a first version of this study.

Author contributions DR and LA conceived the ideas and designed the methodology; LA, FF, FC, FDB and BP collected the data; FF and LA analysed the data; DR, FF and LA led the 
writing of the manuscript; all authors contributed critically to the drafts and gave final approval for publication.

Funding Open Access funding provided by Università degli Studi di Napoli Federico II. This study received no specific funding; BP was funded by and Erasmus Grant KA107.

Data availability The dataset generated and analysed during the current study are available by contacting the first authors, upon reasonable request.

\section{Compliance with ethical standards}

Conflict of interest The authors declare that they have no conflict of interest.

Open Access This article is licensed under a Creative Commons Attribution 4.0 International License, which permits use, sharing, adaptation, distribution and reproduction in any medium or format, as long as you give appropriate credit to the original author(s) and the source, provide a link to the Creative Commons licence, and indicate if changes were made. The images or other third party material in this article are included in the article's Creative Commons licence, unless indicated otherwise in a credit line to the material. If material is not included in the article's Creative Commons licence and your intended use is not permitted by statutory regulation or exceeds the permitted use, you will need to obtain permission directly from the copyright holder. To view a copy of this licence, visit http://creativecommons.org/licenses/by/4.0/.

\section{References}

Alkemade R, Reid RS, Van Den Berg M et al (2013) Assessing the impacts of livestock production on biodiversity in rangeland ecosystems. Proc Natl Acad Sci USA 110:20900-20905

Ancillotto L, Ariano A, Nardone V et al (2017) Effects of freeranging cattle and landscape complexity on bat foraging: Implications for bat conservation and livestock management. Agric Ecosyst Environ 241:54-61

Ancillotto L, Bosso L, Salinas-Ramos VB, Russo D (2019) The importance of ponds for the conservation of bats in urban landscapes. Landsc Urban Plan 190:103607

Bates D, Maechler M, Bolker B, et al (2020) Package Lme4.

Bellamy C, Scott C, Altringham J (2013) Multiscale, presenceonly habitat suitability models: fine-resolution maps for eight bat species. J Appl Ecol 50:892-901

Benton TG, Vickery JA, Wilson JD (2003) Farmland biodiversity: Is habitat heterogeneity the key? Trends Ecol Evol 18:182-188

Böhm SM, Wells K, Kalko EKV (2011) Top-down control of herbivory by birds and bats in the canopy of temperate broad-leaved oaks (Quercus robur). PLoS ONE 6:e17857

Boyles JG, Cryan PM, McCracken GF, Kunz TH (2011) Economic importance of bats in agriculture. Science 332:41-42
Bretz F, Westfall P, Heiberger RM, et al (2020) Package ‘ multcomp,

Crome F, Richards G (1988) Bats and gaps: microchiropteran community structure in a Queensland rain forest. Ecol Soc Am 69:1960-1969

De Conno C, Nardone V, Ancillotto L et al (2018) Testing the performance of bats as indicators of riverine ecosystem quality. Ecol Indic 95:741-750

Downs NC, Racey PA (2006) The use by bats of habitat features in mixed farmland in Scotland. Acta Chiropterol $8: 169-185$

Downs NC, Sanderson LJ (2010) Do bats forage over cattle dung or over cattle? Acta Chiropterol 12:349-358

Dunn J, Waters D (2012) Altitudinal effects on habitat selection in two sympatric pipistrelle species. Mammalia 76:427-433

Entwistle AG (1996) Habitat exploitation by a gleaning bat, Plecotus auritus. Philos Trans R Soc B Biol Sci 351:921-931

Ferreira AS, Peres CA, Bogoni JA, Cassano CR (2018) Use of agroecosystem matrix habitats by mammalian carnivores (Carnivora): a global-scale analysis. Mamm Rev 48:312-327

Fraser GS, Stutchbury BJM (2004) Area-sensitive forest birds move extensively among forest patches. Biol Conserv 118:377-387

Frey-Ehrenbold A, Bontadina F, Arlettaz R, Obrist MK (2013) Landscape connectivity, habitat structure and activity of bat guilds in farmland-dominated matrices. J Appl Ecol 50:252-261

Frick WF, Kingston T, Flanders J (2019) A review of the major threats and challenges to global bat conservation. Ann N Y Acad Sci.

Froidevaux JSP, Boughey KL, Hawkins CL et al (2019) Managing hedgerows for nocturnal wildlife: Do bats and their insect prey benefit from targeted agri-environment schemes? J Appl Ecol 56:1610-1623

Georgiakakis P, Vasilakopoulos P, Mylonas M, Russo D (2010) Bat species richness and activity over an elevation gradient in Mediterranean shrublands of Crete. Hystrix 21:43-56

Gonçalves P, Alcobia S, Simões L, Santos-Reis M (2012) Effects of management options on mammal richness in a Mediterranean agro-silvo-pastoral system. Agrofor Syst 85:383-395

Griffin DR, Webster FA, Michael CR (1960) The echolocation of flying insects by bats. Anim Behav 8:141-154

Grindal SD, Brigham RM (1999) Impacts of forest harvesting on habitat use by foraging insectivorous bats at different spatial scales. Ecoscience 6:25-34

Grindal SD, Morissette JL, Brigham RM (1999) Concentration of bat activity in riparian habitats over an elevational gradient. Can J Zool 77:972-977

Hearn R (2015) Gains and losses in the European mammal fauna. Eur Chang Woods For From Wildwood to Manag Landscapes 193-206

Heim O, Treitler JT, Tschapka M et al (2015) The importance of Landscape elements for bat activity and species richness in agricultural areas. PLoS ONE 10:1-13

Jaberg C, Guisan A (2001) Modelling the distribution of bats in relation to landscape structure in a temperate mountain environment. J Appl Ecol 38:1169-1181 
Jones G, Jacobs DS, Kunz TH et al (2009) Carpe noctem: the importance of bats as bioindicators. Endanger Species Res 8:93-115

Jung M (2013) LecoS-A QGIS plugin for automated landscape ecology analysis (No. e116v2). PeerJ PrePrints.

Kervyn T, Godin MC, Jocqué R et al (2012) Web-building spiders and blood-feeding flies as prey of the notch-eared bat (Myotis emarginatus). Belgian J Zool 142:59-67

Klingbeil BT, Willig MR (2009) Guild-specific responses of bats to landscape composition and configuration in fragmented Amazonian rainforest. J Appl Ecol 46:203-213

Krull D, Schumm A, Metzner W, Neuweiler G (1991) Foraging areas and foraging behavior in the notch-eared bat, Myotis emarginatus (Vespertilionidae). Behav Ecol Sociobiol 28:247-253

Kusch J, Weber C, Idelberger S, Koob T (2004) Foraging habitat preferences of bats in relation to food supply and spatial vegetation structures in a western European low mountain range forest. Folia Zool 53:113-128

Limpens HJGA, Kapteyn K (1991) Bats, their behavior and linear landscape elements. Myotis 29:39-48

Lison F, Jimenez-Franco MV, Altamirano A et al (2020) Bat ecology and conservation in semi-arid and arid landscapes: a globalsystematic review. Mamm Rev 50:52-67

Mcadam JH, Sibbald AR, Teklehaimanot Z, Eason WR (2007) Developing silvopastoral systems and their effects on diversity of fauna. Agrofor Syst 70:81-89

Merritt RW, Anderson JR (1977). The effects of different pasture and rangeland ecosystems on the annual dynamics of insects in cattle droppings. University of California Division of Agricultural Sciences

Morris AD, Miller DA, Kalcounis-Rueppell MC (2010) Use of forest edges by bats in a managed pine forest landscape. J Wildl Manag 74:26-34

Norberg UM, Rayner JM (1987) Ecological morphology and flight in bats (Mammalia; Chiroptera): wing adaptations, flight performance, foraging strategy and echolocation. Philos Trans Royal Soc London B Biol Sci 316(1179):335-427

Palmer MS, Krueger J, Isbell F (2019) Bats join the ranks of oxpeckers and cleaner fish as partners in a pest-reducing mutualism. Ethology 125:170-175

Park KJ (2015) Mitigating the impacts of agriculture on biodiversity: bats and their potential role as bioindicators. Mamm Biol 80(3):191-204

Pereira HM, Leadley PW, Proença V et al (2010) Scenarios for global biodiversity in the 21 st century. Science 330:1496-1501

Roiz D, Neteler M, Castellani C et al (2011) Climatic factors driving invasion of the tiger mosquito (Aedes albopictus) into new areas of Trentino, Northern Italy. PLoS ONE $6: 4-11$

Russo D, Jones G (2002) Identification of twenty-two bat species (Mammalia: Chiroptera) from Italy by analysis of time-expanded recordings of echolocation calls. J Zool 258:91-103

Russo D, Cistrone L, Jones G (2007) Emergence time in forest bats: the influence of canopy closure. Acta Oecol 31:119-126

Russo D, Ancillotto L, Cistrone L, Korine C (2016) The buzz of drinking on the wing in echolocating bats. Ethology 122:226-235

Russo D, Ancillotto L, Hughes AC et al (2017a) Collection of voucher specimens for bat research: conservation, ethical implications, reduction, and alternatives. Mamm Rev 47:237-246

Russo D, Cistrone L, Budinski I,et al, (2017b) Sociality influences thermoregulation and roost switching in a forest bat using ephemeral roosts. Ecol Evol 7:5310-5321

Russo D, Ancillotto L, Jones G (2018a) Bats are still not birds in the digital era: echolocation call variation and why it matters for bat species identification. Can J Zool 96:63-78

Russo D, Bosso L, Ancillotto L (2018b) Novel perspectives on bat insectivory highlight the value of this ecosystem service in farmland: Research frontiers and management implications. Agric Ecosyst Environ 266:31-38

Salinas-Ramos VB, Ancillotto L, Bosso L, Sánchez-Cordero V, Russo D (2020) Interspecific competition in bats: state of knowledge and research challenges. Mammal Rev 50(1):68-81

Santini L, González-Suárez M, Russo D et al (2019) One strategy does not fit all: determinants of urban adaptation in mammals. Ecol Lett 22:365-376

Siemers BM, Kriner E, Kaipf I et al (2012) Bats eavesdrop on the sound of copulating flies. Curr Biol 22:R563-R564

Sokos CK, Mamolos AP, Kalburtji KL, Birtsas PK (2013) Farming and wildlife in Mediterranean agroecosystems. J Nat Conserv 21:81-92

Standfast HA, Dyce AL (1968) Attacks on cattle by mosquitoes and biting midges. Aust Vet J 44(12):585

Taylor LR (1963) Analysis of the effect of temperature on insects in flight. Br Ecol Soc 32:99-117

Voigt CC, Holderied MW (2012) High manoeuvring costs force narrow-winged molossid bats to forage in open space. J Comp Physiol B Biochem Syst Environ Physiol 182:415-424

Wickramasinghe LP, Harris S, Jones G, Vaughan N (2003) Bat activity and species richness on organic and conventional farms: Impact of agricultural intensification. J Appl Ecol 40:984-993

Zuur AF, Ieno EN, Elphick CS (2010) A protocol for data exploration to avoid common statistical problems. Methods Ecol Evol 1:3-14

Publisher's Note Springer Nature remains neutral with regard to jurisdictional claims in published maps and institutional affiliations. 